\title{
[(1-Methylcytosine)2H]I, an asymmetric base pair
}

Müller, Jens ; Freisinger, Eva

\begin{abstract}
The X-ray crystal structure of the title compound, 1-methylcytosinium iodide 1-methylcytosine, $[(1-\mathrm{MeC}) 2 \mathrm{H}] \mathrm{I}(1-\mathrm{MeC}$ is 1 -methylcytosine $)$ or $\mathrm{C} 5 \mathrm{H} 8 \mathrm{~N} 3 \mathrm{O}+\cdot \cdot \mathrm{I}-\cdot \mathrm{C} 5 \mathrm{H} 7 \mathrm{~N} 3 \mathrm{O}$, has been determined at 163 $\mathrm{K}$. In this compound, one protonated (1-MeCH+) and one neutral methylcytosine (1-MeC) moiety form an asymmetric base pair comprising three hydrogen bonds. A previous structure determination [Krüger, Bruhn Steinborn (2004). Org. Biomol. Chem.2, 2513-2516] showed the same cell parameters but a centrosymmetric base pair in space group P21/c. At low temperature, however, we discovered the title compound to be a racemic twin showing pseudo-centrosymmetry.
\end{abstract}

DOI: https://doi.org/10.1107/S1600536805000693

Posted at the Zurich Open Repository and Archive, University of Zurich ZORA URL: https://doi.org/10.5167/uzh-59913

Journal Article

Published Version

Originally published at:

Müller, Jens; Freisinger, Eva (2005). [(1-Methylcytosine)2H]I, an asymmetric base pair. Acta Crystallographica Section E Structure Reports Online, 61(2):o320-o322.

DOI: https://doi.org/10.1107/S1600536805000693 
Acta Crystallographica Section E

Structure Reports

Online

ISSN 1600-5368

\section{[(1-Methylcytosine $\left.)_{2} \mathrm{H}\right] \mathrm{I}$, an asymmetric base pair}

Jens Müller $^{\mathrm{a} *}$ and Eva Freisinger ${ }^{\mathrm{b}}$

a University of Dortmund, Inorganic Chemistry, Otto-Hahn-Straße 6, 44227 Dortmund, Germany, and ${ }^{\mathbf{b}}$ University of Zürich, Institute of Inorganic Chemistry, Winterthurerstrasse 190, 8057 Zürich, Switzerland

Correspondence e-mail: mueller@muellerlab.org

\section{Key indicators}

Single-crystal X-ray study

$T=163 \mathrm{~K}$

Mean $\sigma(\mathrm{C}-\mathrm{C})=0.005 \AA$

$R$ factor $=0.020$

$w R$ factor $=0.043$

Data-to-parameter ratio $=13.8$

For details of how these key indicators were automatically derived from the article, see http://journals.iucr.org/e.
The X-ray crystal structure of the title compound, 1-methylcytosinium iodide 1-methylcytosine, $\left[(1-\mathrm{MeC})_{2} \mathrm{H}\right] \mathrm{I}$ (1-MeC is 1-methylcytosine) or $\mathrm{C}_{5} \mathrm{H}_{8} \mathrm{~N}_{3} \mathrm{O}^{+} \cdot \mathrm{I}^{-} \cdot \mathrm{C}_{5} \mathrm{H}_{7} \mathrm{~N}_{3} \mathrm{O}$, has been determined at $163 \mathrm{~K}$. In this compound, one protonated $\left(1-\mathrm{MeCH}^{+}\right)$and one neutral methylcytosine (1-MeC) moiety form an asymmetric base pair comprising three hydrogen bonds. A previous structure determination [Krüger, Bruhn \& Steinborn (2004). Org. Biomol. Chem. 2, 2513-2516] showed the same cell parameters but a centrosymmetric base pair in space group $P 2_{1} / c$. At low temperature, however, we discovered the title compound to be a racemic twin showing pseudo-centrosymmetry.

\section{Comment}

Hydrogen bonds are a common structural feature in nucleic acids (Lavery \& Zakrzewska, 1999). Although typically two different nucleobases undergo base pairing (e.g. guanine:cytosine, adenine:thymine), several hydrogen-bonding patterns are known with base pairing between identical nucleobases. The i-motif, consisting of two parallel-stranded intertwined double helices made up entirely of cytosine, is a well known example (Patel et al., 1999). A recent publication describes the crystal structure of a centrosymmetric hemiprotonated methylcytosine:methylcytosine base pair, $\left[(1-\mathrm{MeC})_{2} \mathrm{H}\right] \mathrm{I}$ (Krüger et al., 2004). Concomitant DFT calculations suggest the formation of an asymmetric base pair, however. We report here the crystal structure of this compound in a different crystal configuration, determined at $163 \mathrm{~K}$, displaying the theoretically predicted asymmetric base pairing pattern (Fig. 1). The crystal was obtained in the course of our studies of model systems for metal-mediated base pairs (Müller et al., 2005).
Received 4 January 2005 Accepted 10 January 2005 Online 22 January 2005

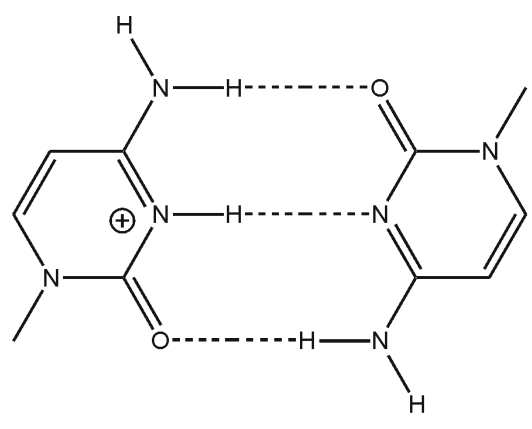

(I)

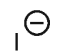




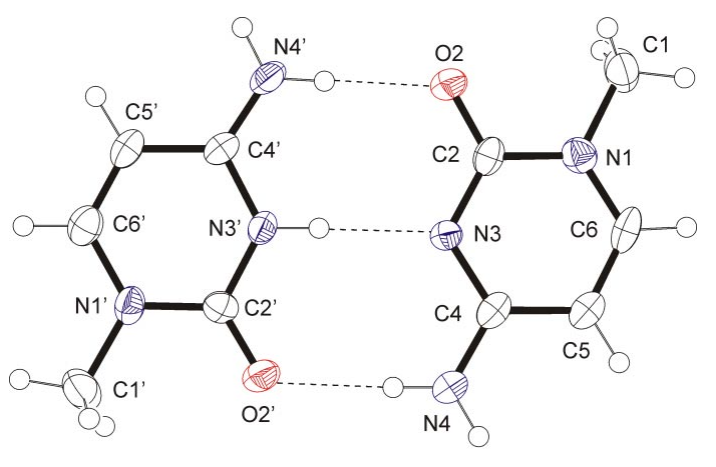

11

Figure 1

View of the cation of the title compound with the atom numbering scheme. Displacement ellipsoids are drawn at the $50 \%$ probability level.

The hydrogen-bonded nucleobases of the title compound are almost coplanar $\left[1.6(2)^{\circ}\right]$. Two base pairs are bridged via the iodide counter-ion (Fig. 2), which forms hydrogen bonds to the exocyclic amine groups of both bases [3.633 (2) and 3.717 (3) §]. Two crystallographically independent $1-\mathrm{MeC}$ moieties of neighbouring base pairs stack at a distance of approximately $3.4 \AA$, leading to the formation of a chain structure with two individual chains I and II (Fig. 3).

The $\mathrm{H}$ atom at the $\mathrm{N} 3$ position of $1-\mathrm{MeCH}^{+}$was located in difference Fourier maps. The protonation site is further corroborated by an increase in the $\mathrm{C} 2-\mathrm{N} 3-\mathrm{C} 4$ angle [125.1 (3) ${ }^{\circ}$ in $1-\mathrm{MeCH}^{+}$and $119.6(2)^{\circ}$ in $1-\mathrm{MeC}$. In addition, the two $\mathrm{N} 4(\mathrm{H}) \cdots \mathrm{O}$ hydrogen bonds display different bond lengths, resulting in a 2.762 (4) $\AA$ distance for the stronger bond involving $\mathrm{N} 4(\mathrm{H})$ of $1-\mathrm{MeCH}^{+}$and 2.901 (3) $\AA$ for the weaker bond (see Table 2 for hydrogen-bond geometries). Accordingly, both the $\mathrm{C} 4-\mathrm{N} 4$ and the $\mathrm{C} 2-\mathrm{O} 2$ bonds are significantly shorter in $1-\mathrm{MeCH}^{+}$than in $1-\mathrm{MeC}[1.291$ (5) versus 1.333 (4) $\AA$ and 1.219 (3) versus 1.245 (3) $\AA$ ]. The $\mathrm{N} 3 \cdots \mathrm{N} 3^{\prime}$ distance is $2.808(4) \AA$. All these experimental values correlate well with those of the DFT study (Krüger et al., 2004).

A comparison of the two different crystal forms of $\left[(1-\mathrm{MeC})_{2} \mathrm{H}\right] \mathrm{I}$ with related structures of hemiprotonated cytosine dimers containing a variety of other counter-ions or solvent molecules reveals that in almost all cases asymmetric base pairs are observed (Salam \& Aoki, 2000; Schimanski et al., 1998; Kistenmacher et al., 1980; Fujinami et al., 1979; Kistenmacher et al., 1979). Only one of the crystallographically independent $1-\mathrm{MeCH}^{+}: 1-\mathrm{MeC}$ pairs in the structure of cytosine hemitrichloroacetate has been proposed as truly symmetric (Gdaniec et al., 1988), whereas in the other centrosymmetric structures known to date this symmetry appears to be a consequence of a disordered $\mathrm{H}$ atom in the central hydrogen bond (Krüger et al., 2004; Gdaniec et al., 1988). The difference between the crystal structure presented in this work and its symmetric counterpart (Krüger et al., 2004) is presumably based on a temperature-dependent change from disorder to order, supporting this assumption.

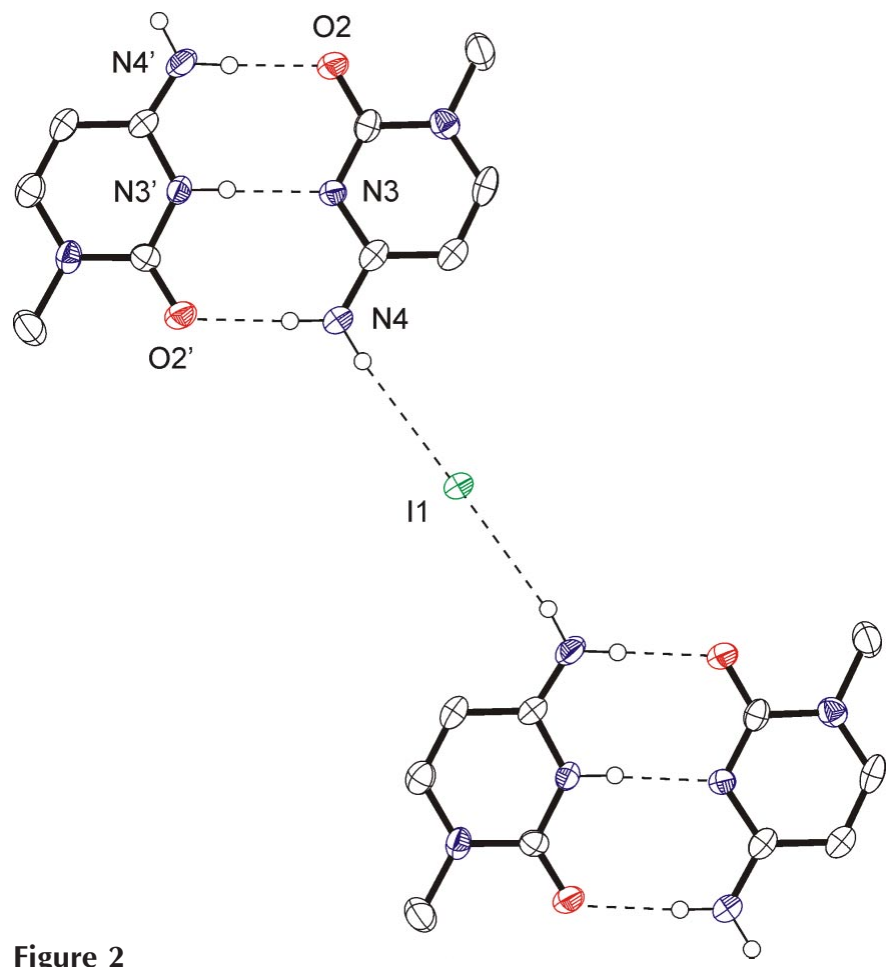

Two base pairs bridged via an iodide counter-ion. Displacement ellipsoids are drawn at the $50 \%$ probability level. The unlabelled asymmetric pair is related by the symmetry operator $(x+1, y, z+1)$. Dashed lines indicate hydrogen bonds.

\section{Experimental}

The title compound was synthesized according to published protocols (Kistenmacher et al., 1979).

Crystal data

$\mathrm{C}_{5} \mathrm{H}_{8} \mathrm{~N}_{3} \mathrm{O}^{+} \cdot \mathrm{I}^{-} \cdot \mathrm{C}_{5} \mathrm{H}_{7} \mathrm{~N}_{3} \mathrm{O}$

$M_{r}=378.18$

Monoclinic, $P 2_{1}$

$a=7.195(1) \AA$

$b=8.629(2) \AA$

$c=11.522(2) \AA$

$\beta=97.59(3)$

$V=709.1(2) \AA^{3}$

$Z=2$

\author{
$D_{x}=1.771 \mathrm{Mg} \mathrm{m}^{-3}$ \\ Mo $K \alpha$ radiation \\ Cell parameters from 4452 \\ reflections \\ $\theta=2.9-25.4^{\circ}$ \\ $\mu=2.27 \mathrm{~mm}^{-1}$ \\ $T=163$ (2) K \\ Block, colourless \\ $0.50 \times 0.40 \times 0.38 \mathrm{~mm}$
}

\section{Data collection}

Nonius KappaCCD diffractometer

$\omega$ scans

Absorption correction: multi-scan (SCALEPACK; Otwinowski \& Minor, 1997)

$T_{\min }=0.358, T_{\max }=0.427$

4462 measured reflections

\section{Refinement}

Refinement on $F^{2}$

$R\left[F^{2}>4 \sigma\left(F^{2}\right)\right]=0.020$

$w R\left(F^{2}\right)=0.043$

$S=0.98$

2519 reflections

183 parameters

$\mathrm{H}$ atoms treated by a mixture of independent and constrained refinement
2519 independent reflections 2291 reflections with $I>2 \sigma(I)$

$R_{\text {int }}=0.022$

$\theta_{\text {max }}=25.4^{\circ}$

$h=-8 \rightarrow 8$

$k=-10 \rightarrow 10$

$l=-13 \rightarrow 13$

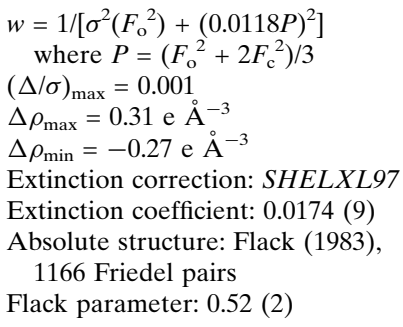


Table 1

Selected geometric parameters $\left(\AA{ }^{\circ}{ }^{\circ}\right)$.

\begin{tabular}{llll}
\hline $\mathrm{C} 2-\mathrm{O} 2$ & $1.245(3)$ & $\mathrm{C} 2^{\prime}-\mathrm{O} 2^{\prime}$ & $1.219(3)$ \\
$\mathrm{C} 2-\mathrm{N} 3$ & $1.359(4)$ & $\mathrm{C} 2^{\prime}-\mathrm{N} 3^{\prime}$ & $1.368(4)$ \\
$\mathrm{N} 3-\mathrm{C} 4$ & $1.342(3)$ & $\mathrm{N} 3^{\prime}-\mathrm{C} 4^{\prime}$ & $1.355(3)$ \\
$\mathrm{C} 4-\mathrm{N} 4$ & $1.333(4)$ & $\mathrm{C} 4^{\prime}-\mathrm{N} 4^{\prime}$ & $1.291(5)$ \\
& & & \\
$\mathrm{O} 2-\mathrm{C} 2-\mathrm{N} 3$ & $122.3(3)$ & $\mathrm{O} 2^{\prime}-\mathrm{C} 2^{\prime}-\mathrm{N} 3^{\prime}$ & $121.8(3)$ \\
$\mathrm{O} 2-\mathrm{C} 2-\mathrm{N} 1$ & $118.6(3)$ & $\mathrm{O} 2^{\prime}-\mathrm{C} 2^{\prime}-\mathrm{N} 1^{\prime}$ & $121.7(4)$ \\
$\mathrm{N} 3-\mathrm{C} 2-\mathrm{N} 1$ & $119.2(3)$ & $\mathrm{N} 3^{\prime}-\mathrm{C} 2^{\prime}-\mathrm{N} 1^{\prime}$ & $116.5(3)$ \\
$\mathrm{C} 4-\mathrm{N} 3-\mathrm{C} 2$ & $119.6(2)$ & $\mathrm{C} 4^{\prime}-\mathrm{N} 3^{\prime}-\mathrm{C} 2^{\prime}$ & $125.1(3)$ \\
$\mathrm{N} 4-\mathrm{C} 4-\mathrm{N} 3$ & $117.9(3)$ & $\mathrm{N} 4^{\prime}-\mathrm{C} 4^{\prime}-\mathrm{N}^{\prime}$ & $119.7(3)$ \\
$\mathrm{N} 4-\mathrm{C} 4-\mathrm{C} 5$ & $119.9(2)$ & $\mathrm{N} 4^{\prime}-\mathrm{C} 4^{\prime}-\mathrm{C}^{\prime}$ & $123.2(3)$ \\
$\mathrm{N} 3-\mathrm{C} 4-\mathrm{C} 5$ & $122.2(3)$ & $\mathrm{N} 3^{\prime}-\mathrm{C} 4^{\prime}-\mathrm{C}^{\prime}$ & $117.0(3)$ \\
\hline
\end{tabular}

Table 2

Hydrogen-bond geometry $\left(\AA{ }^{\circ}\right)$.

\begin{tabular}{lllll}
\hline$D-\mathrm{H} \cdots A$ & $D-\mathrm{H}$ & $\mathrm{H} \cdots A$ & $D \cdots A$ & $D-\mathrm{H} \cdots A$ \\
\hline $\mathrm{N}^{\prime}-\mathrm{H}^{\prime} \cdots \mathrm{N} 3$ & $0.82(4)$ & $1.99(4)$ & $2.808(4)$ & $171(4)$ \\
$\mathrm{N}^{\prime}-\mathrm{H} 44^{\prime} 1 \cdots \mathrm{O} 2$ & 0.86 & 1.92 & $2.762(4)$ & 166 \\
$\mathrm{~N} 4-\mathrm{H} 4 A \cdots \mathrm{O} 2$ & 0.86 & 2.07 & $2.901(3)$ & 161 \\
$\mathrm{~N} 4-\mathrm{H} 4 B \cdots \mathrm{I} 1$ & 0.86 & 2.87 & $3.717(2)$ & 169 \\
$\mathrm{~N}^{\prime}-\mathrm{H} 4{ }^{\prime} 2 \cdots \mathrm{I} 1^{\mathrm{i}}$ & 0.86 & 2.78 & $3.633(2)$ & 171 \\
$\mathrm{C} 5-\mathrm{H} 5 \cdots \mathrm{O} 2^{\prime i i}$ & 0.93 & 2.47 & $3.088(4)$ & 124 \\
$\mathrm{C}^{\prime}-\mathrm{H} 5^{\prime} \cdots \mathrm{O} 2^{\text {iii }}$ & 0.93 & 2.48 & $3.085(4)$ & 123
\end{tabular}

Symmetry codes: (i) $x-1, y, z-1$; (ii) $-x+1, y+\frac{1}{2},-z+1$; (iii) $-x, y-\frac{1}{2},-z$.

$\mathrm{H}$ atoms, except $\mathrm{H}^{\prime}$, were included in calculated positions with distances ranging from 0.86 to $0.96 \AA$ and included in the refinement in the riding-model approximation with refined isotropic displacement parameters. Atom $\mathrm{H}^{\prime}{ }^{\prime}$ was found in the difference Fourier map and refined without restraints.

Data collection: KappaCCD Software (Nonius, 1998); cell refinement: DENZO and SCALEPACK (Otwinowski \& Minor, 1997); data reduction: $D E N Z O$ and $S C A L E P A C K$; program(s) used to solve structure: SHELXS86 (Sheldrick, 1990a); program(s) used to refine structure: SHELXL97 (Sheldrick, 1997); molecular graphics: SHELXTL-Plus (Sheldrick, 1990b); software used to prepare material for publication: SHELXL97.

This work was supported by the Deutsche Forschungsgemeinschaft (Emmy Noether-Programme), the Department of Chemistry at the University of Dortmund and the Fonds der Chemischen Industrie. The authors thank Professor Dr Bernhard Lippert for his continuous support.

\section{References}

Flack, H. D. (1983). Acta Cryst. A39, 876-881.

Fujinami, F., Ogawa, K., Arakawa, Y., Shirotake, S., Fuji, S. \& Tomita, K.-I. (1979). Acta Cryst. B35, 968-970.

Gdaniec, M., Brycki, B. \& Szafran, M. (1988). J. Chem. Soc. Perkin Trans. 2, pp. $1775-1779$.

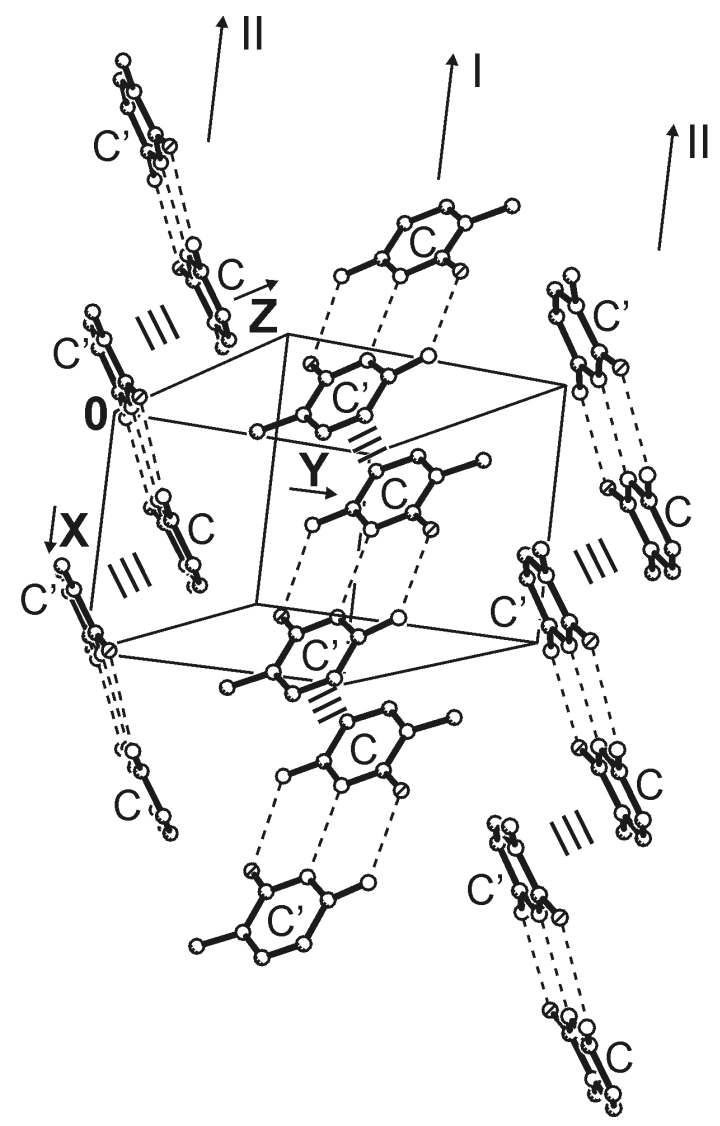

Figure 3

Chain structure formed by base stacking of crystallographically independent 1-MeC moieties. Dashed lines indicate hydrogen bonds.

Kistenmacher, T. J., Rossi, M., Caradonna, J. P. \& Marzilli, L. G. (1979). $A d v$. Mol. Relax. Interact. Proc. 15, 119-133.

Kistenmacher, T. J., Rossi, M., Chiang, C. C., Caradonna, J. P. \& Marzilli, L. (1980). Adv. Mol. Relax. Interact. Proc. 17, 113-134.

Krüger, T., Bruhn, C. \& Steinborn, D. (2004). Org. Biomol. Chem. 2, 25132516.

Lavery, R. \& Zakrzewska, K. (1999). Oxford Handbook of Nucleic Acid Structure, edited by S. Neidle, pp. 39-76. New York: Oxford University Press Inc.

Müller, J., Polonius, F.-A. \& Roitzsch, M. (2005). Inorg. Chim. Acta. In the press (doi: 10.1016/j.ica.2004.11.038).

Nonius (1998). KappaCCD Software. Nonius BV, Delft, The Netherlands.

Otwinowski, Z. \& Minor, W. (1997). Methods in Enzymology, Vol. 276, Macromolecular Crystallography, Part A, edited by C. W. Carter Jr \& R. M. Sweet, pp. 307-326. New York: Academic Press.

Patel, D. J., Bouaziz, S., Kettani, A. \& Wang, Y. (1999). Oxford Handbook of Nucleic Acid Structure, edited by S. Neidle, pp. 389-453. New York: Oxford University Press Inc.

Salam, M. A. \& Aoki, K. (2000). Inorg. Chim. Acta, 311, 15-24.

Schimanski, A., Freisinger, E., Erxleben, A. \& Lippert, B. (1998). Inorg. Chim. Acta, 283, 223-232.

Sheldrick, G. M. (1990a). Acta Cryst. A46, 467-473.

Sheldrick, G. M. (1990b). SHELXTL-Plus (VMS). Siemens Analytical X-ray Instruments Inc., Madison, Wisconsin, USA.

Sheldrick, G. M. (1997). SHELXL97. University of Göttingen, Germany. 


\section{supporting information}

Acta Cryst. (2005). E61, o320-o322 [https://doi.org/10.1107/S1600536805000693]

\section{[(1-Methylcytosine $\left.)_{2} \mathrm{H}\right] \mathrm{I}$, an asymmetric base pair}

Jens Müller and Eva Freisinger

1-Methylcytosinium iodide 1-methylcytosine

Crystal data

$\mathrm{C}_{5} \mathrm{H}_{8} \mathrm{~N}_{3} \mathrm{O}^{+} \cdot \mathrm{I}^{-\cdot} \cdot \mathrm{C}_{5} \mathrm{H}_{7} \mathrm{~N}_{3} \mathrm{O}$

$M_{r}=378.18$

Monoclinic, $P 2_{1}$

Hall symbol: $\mathrm{P} 2 \mathrm{yb}$

$a=7.195$ (1) $\AA$

$b=8.629(2) \AA$

$c=11.522(2) \AA$

$\beta=97.59(3)^{\circ}$

$V=709.1(2) \AA^{3}$

$Z=2$

\section{Data collection}

Nonius KappaCCD diffractometer

Radiation source: fine-focus sealed tube

Graphite monochromator

Detector resolution: 44 pixels $\mathrm{mm}^{-1}$

360 frames via $\omega$-rotation $\left(\Delta \omega \overline{1}^{\circ}\right)$ and two times $5 \mathrm{~s}$ per frame scans

Absorption correction: multi-scan (SCALEPACK; Otwinowski \& Minor, 1997)

Refinement

Refinement on $F^{2}$

Least-squares matrix: full

$R\left[F^{2}>2 \sigma\left(F^{2}\right)\right]=0.020$

$w R\left(F^{2}\right)=0.043$

$S=0.98$

2519 reflections

183 parameters

1 restraint

Primary atom site location: structure-invariant direct methods

Secondary atom site location: difference Fourier map

Hydrogen site location: inferred from neighbouring sites
$F(000)=372$

$D_{\mathrm{x}}=1.771 \mathrm{Mg} \mathrm{m}^{-3}$

Mo $K \alpha$ radiation, $\lambda=0.71069 \AA$

Cell parameters from 4452 reflections

$\theta=2.9-25.4^{\circ}$

$\mu=2.27 \mathrm{~mm}^{-1}$

$T=163 \mathrm{~K}$

Block, colourless

$0.50 \times 0.40 \times 0.38 \mathrm{~mm}$

$T_{\min }=0.358, T_{\max }=0.427$

4435 measured reflections

2519 independent reflections

2291 reflections with $I>2 \sigma(I)$

$R_{\text {int }}=0.022$

$\theta_{\text {max }}=25.4^{\circ}, \theta_{\min }=2.9^{\circ}$

$h=-8 \rightarrow 8$

$k=-10 \rightarrow 10$

$l=-13 \rightarrow 13$

$\mathrm{H}$ atoms treated by a mixture of independent and constrained refinement

$w=1 /\left[\sigma^{2}\left(F_{\mathrm{o}}^{2}\right)+(0.0118 P)^{2}\right]$ where $P=\left(F_{\mathrm{o}}^{2}+2 F_{\mathrm{c}}^{2}\right) / 3$

$(\Delta / \sigma)_{\max }=0.001$

$\Delta \rho_{\max }=0.31$ e $\AA^{-3}$

$\Delta \rho_{\min }=-0.27$ e $\AA^{-3}$

Extinction correction: SHELXL97, $\mathrm{Fc}^{*}=\mathrm{kFc}\left[1+0.001 \times \mathrm{Fc}^{2} \lambda^{3} / \sin (2 \theta)\right]^{-1 / 4}$

Extinction coefficient: 0.0174 (9)

Absolute structure: Flack (1983), 1166 Friedel pairs

Absolute structure parameter: 0.52 (2) 


\section{Special details}

Geometry. All e.s.d.'s (except the e.s.d. in the dihedral angle between two l.s. planes) are estimated using the full covariance matrix. The cell e.s.d.'s are taken into account individually in the estimation of e.s.d.'s in distances, angles and torsion angles; correlations between e.s.d.'s in cell parameters are only used when they are defined by crystal symmetry. An approximate (isotropic) treatment of cell e.s.d.'s is used for estimating e.s.d.'s involving 1.s. planes.

Refinement. Intensity data were collected on an Enraf-Nonius KappaCCD (Nonius BV, Netherlands) (Mo K $\alpha, \lambda=$ $0.71069 \AA$, graphite-monochromator). The structure was solved by standard Patterson methods (Sheldrick 1990a) and refined by full-matrix least-squares based on $F^{2}$ using the SHELXTL-Plus (Sheldrick 1990b) and SHELXL97 (Sheldrick 1997) programs. The positions of all non-hydrogen atoms were deduced from difference Fourier maps and refined anisotropically. Initially, the Flack parameter was refined to a value of 0.51 (2) with:_refine_ls_R_factor_all 0.0263 _refine_ls_R_factor_gt 0.0220 _refine_ls_wR_factor_ref 0.0493 _refine_ls_wR_factor_gt 0.0485 and _refine_ls_goodness_of_fit_ref 1.040 Accordingly, subsequent application of the twin law $\left(\begin{array}{lllllllll}-1 & 0 & 0, & 0 & -1 & 0, & 0 & 0 & -1\end{array}\right)$ resulted in a racemic twin fraction of 0.51881 with the $R$ factors presented below. Refinement of $F^{2}$ against ALL reflections. The weighted $R$-factor $w R$ and goodness of fit $S$ are based on $F^{2}$, conventional $R$-factors $R$ are based on $F$, with $F$ set to zero for negative $F^{2}$. The threshold expression of $F^{2}>\sigma\left(F^{2}\right)$ is used only for calculating $R$-factors(gt) etc. and is not relevant to the choice of reflections for refinement. $R$-factors based on $F^{2}$ are statistically about twice as large as those based on $F$, and $R$ - factors based on ALL data will be even larger.

Fractional atomic coordinates and isotropic or equivalent isotropic displacement parameters $\left(\AA^{2}\right)$

\begin{tabular}{|c|c|c|c|c|}
\hline & $x$ & $y$ & $z$ & $U_{\text {iso }} * / U_{\text {eq }}$ \\
\hline $\mathrm{H} 3^{\prime}$ & $0.163(5)$ & $-0.025(5)$ & $0.248(3)$ & $0.045(10)^{*}$ \\
\hline I1 & $0.75919(2)$ & $0.00071(13)$ & $0.754516(15)$ & $0.03646(9)$ \\
\hline N1 & $0.6507(4)$ & $0.2149(4)$ & 0.2055 & $0.0297(9)$ \\
\hline $\mathrm{C} 1$ & $0.7037(5)$ & $0.3110(5)$ & $0.1102(3)$ & $0.0419(10)$ \\
\hline $\mathrm{H} 1 \mathrm{~A}$ & 0.7033 & 0.2488 & 0.0410 & $0.048(4)^{*}$ \\
\hline H1B & 0.8270 & 0.3526 & 0.1325 & $0.048(4)^{*}$ \\
\hline $\mathrm{H} 1 \mathrm{C}$ & 0.6157 & 0.3943 & 0.0946 & $0.048(4)^{*}$ \\
\hline $\mathrm{C} 2$ & 0.4866 & $0.1298(4)$ & 0.1845 & $0.0261(8)$ \\
\hline $\mathrm{O} 2$ & $0.3983(3)$ & $0.1308(3)$ & $0.08405(16)$ & $0.0308(6)$ \\
\hline N3 & $0.4287(3)$ & $0.0467(3)$ & $0.27326(19)$ & $0.0230(8)$ \\
\hline $\mathrm{C} 4$ & $0.5352(4)$ & $0.0432(4)$ & $0.3781(3)$ & $0.0252(10)$ \\
\hline N4 & $0.4765(3)$ & -0.0428 & $0.4622(2)$ & $0.0305(9)$ \\
\hline $\mathrm{H} 4 \mathrm{~A}$ & 0.3733 & -0.0939 & 0.4484 & $0.046(5)^{*}$ \\
\hline H4B & 0.5418 & -0.0472 & 0.5302 & $0.046(5)^{*}$ \\
\hline $\mathrm{C} 5$ & $0.7054(4)$ & $0.1272(4)$ & $0.4009(3)$ & $0.0313(9)$ \\
\hline $\mathrm{H} 5$ & 0.7789 & 0.1239 & 0.4736 & $0.041(4)^{*}$ \\
\hline C6 & $0.7560(5)$ & $0.2133(5)$ & $0.3103(6)$ & $0.0334(12)$ \\
\hline H6 & 0.8655 & 0.2717 & 0.3218 & $0.041(4)^{*}$ \\
\hline $\mathrm{N} 1^{\prime}$ & $-0.1634(4)$ & $-0.2274(4)$ & $0.2974(4)$ & $0.0290(9)$ \\
\hline $\mathrm{C} 1^{\prime}$ & -0.2220 & $-0.3265(5)$ & $0.3885(3)$ & $0.0434(11)$ \\
\hline $\mathrm{H} 1{ }^{\prime} 1$ & -0.3459 & -0.3653 & 0.3635 & $0.048(4)^{*}$ \\
\hline $\mathrm{H} 1^{\prime} 2$ & -0.2223 & -0.2677 & 0.4592 & $0.048(4)^{*}$ \\
\hline $\mathrm{H} 1^{\prime} 3$ & -0.1364 & -0.4118 & 0.4028 & $0.048(4)^{*}$ \\
\hline $\mathrm{C} 2^{\prime}$ & 0.0013 & $-0.1463(4)$ & 0.3218 & $0.0256(8)$ \\
\hline $\mathrm{O} 2^{\prime}$ & 0.0890 & $-0.1442(3)$ & $0.41984(17)$ & $0.0319(6)$ \\
\hline N3' & $0.0596(4)$ & $-0.0656(3)$ & $0.2310(2)$ & $0.0257(8)$ \\
\hline $\mathrm{C}^{\prime}$ & $-0.0342(4)$ & -0.0578 & 0.1211 & $0.0263(10)$ \\
\hline $\mathrm{N} 4^{\prime}$ & $0.0347(3)$ & $0.0217(6)$ & $0.04203(19)$ & $0.0351(8)$ \\
\hline $\mathrm{H} 4^{\prime} 1$ & 0.1396 & 0.0694 & 0.0592 & $0.046(5)^{*}$ \\
\hline
\end{tabular}




\begin{tabular}{lllll}
$\mathrm{H}^{\prime} 2$ & -0.0244 & 0.0269 & -0.0278 & $0.046(5)^{*}$ \\
$\mathrm{C} 5^{\prime}$ & $-0.2075(4)$ & $-0.1396(4)$ & $0.0995(3)$ & $0.0303(9)$ \\
$\mathrm{H} 5^{\prime}$ & -0.2800 & -0.1352 & 0.0265 & $0.041(4)^{*}$ \\
$\mathrm{C}^{\prime}$ & $-0.2631(5)$ & $-0.2221(5)$ & $0.1857(5)$ & $0.0316(12)$ \\
$\mathrm{H} 6^{\prime}$ & -0.3736 & -0.2789 & 0.1707 & $0.041(4)^{*}$ \\
\hline
\end{tabular}

Atomic displacement parameters $\left(\AA^{2}\right)$

\begin{tabular}{lllllll}
\hline & $U^{11}$ & $U^{22}$ & $U^{33}$ & $U^{12}$ & $U^{13}$ & $U^{23}$ \\
\hline $\mathrm{I} 1$ & $0.03211(11)$ & $0.04901(13)$ & $0.02584(11)$ & $-0.00791(16)$ & $-0.00519(7)$ & $0.00848(13)$ \\
$\mathrm{N} 1$ & $0.0295(16)$ & $0.0269(18)$ & $0.0332(19)$ & $0.0005(16)$ & $0.006(2)$ & $0.0043(18)$ \\
$\mathrm{C} 1$ & $0.0320(16)$ & $0.041(2)$ & $0.053(2)$ & $-0.0079(16)$ & $0.0045(18)$ & $0.010(2)$ \\
$\mathrm{C} 2$ & $0.0184(13)$ & $0.0252(19)$ & $0.0337(18)$ & $0.0049(13)$ & $0.0000(15)$ & $-0.0023(16)$ \\
$\mathrm{O} 2$ & $0.0299(10)$ & $0.0366(14)$ & $0.0247(11)$ & $0.0012(10)$ & $-0.0008(10)$ & $0.0042(11)$ \\
$\mathrm{N} 3$ & $0.0246(11)$ & $0.023(2)$ & $0.0211(11)$ & $0.0006(10)$ & $0.0016(10)$ & $0.0003(11)$ \\
$\mathrm{C} 4$ & $0.0220(13)$ & $0.026(3)$ & $0.0269(15)$ & $0.0071(12)$ & $-0.0003(14)$ & $-0.0073(15)$ \\
$\mathrm{N} 4$ & $0.0298(12)$ & $0.036(2)$ & $0.0244(13)$ & $-0.0013(12)$ & $-0.0026(12)$ & $0.0021(14)$ \\
$\mathrm{C} 5$ & $0.0274(15)$ & $0.035(2)$ & $0.0298(17)$ & $0.0036(15)$ & $-0.0040(15)$ & $-0.0060(18)$ \\
$\mathrm{C} 6$ & $0.018(2)$ & $0.029(2)$ & $0.049(3)$ & $0.0022(19)$ & $-0.008(2)$ & $-0.011(2)$ \\
$\mathrm{N} 1^{\prime}$ & $0.0204(15)$ & $0.0280(19)$ & $0.0368(19)$ & $-0.0002(16)$ & $-0.0030(18)$ & $-0.0009(18)$ \\
$\mathrm{C} 1^{\prime}$ & $0.0327(17)$ & $0.047(3)$ & $0.052(2)$ & $-0.0041(16)$ & $0.0094(18)$ & $0.015(2)$ \\
$\mathrm{C} 2^{\prime}$ & $0.0262(15)$ & $0.0226(19)$ & $0.0279(17)$ & $0.0063(13)$ & $0.0032(15)$ & $0.0038(16)$ \\
$\mathrm{O}^{\prime}$ & $0.0305(10)$ & $0.0371(15)$ & $0.0264(11)$ & $0.0023(10)$ & $-0.0022(10)$ & $0.0046(11)$ \\
$\mathrm{N}^{\prime}$ & $0.0206(11)$ & $0.0295(19)$ & $0.0256(14)$ & $-0.0020(11)$ & $-0.0017(12)$ & $-0.0023(12)$ \\
$\mathrm{C}^{\prime}$ & $0.0258(14)$ & $0.028(3)$ & $0.0232(16)$ & $0.0053(13)$ & $-0.0024(15)$ & $-0.0042(16)$ \\
$\mathrm{N}^{\prime}$ & $0.0292(11)$ & $0.052(2)$ & $0.0220(12)$ & $-0.0027(18)$ & $-0.0041(11)$ & $-0.004(2)$ \\
$\mathrm{C}^{\prime}$ & $0.0224(14)$ & $0.034(2)$ & $0.0318(18)$ & $0.0028(14)$ & $-0.0043(15)$ & $-0.0063(18)$ \\
$\mathrm{C}^{\prime}$ & $0.031(2)$ & $0.026(2)$ & $0.036(3)$ & $0.0009(19)$ & $-0.001(2)$ & $-0.005(2)$ \\
& & & & & & \\
\hline
\end{tabular}

Geometric parameters $\left(\AA,{ }^{\circ}\right)$

\begin{tabular}{llll}
\hline $\mathrm{N} 1-\mathrm{C} 6$ & $1.338(9)$ & $\mathrm{C} 5^{\prime}-\mathrm{C} 6^{\prime}$ & $1.326(6)$ \\
$\mathrm{N} 1-\mathrm{C} 2$ & $1.384(5)$ & $\mathrm{C} 1-\mathrm{H} 1 \mathrm{~A}$ & 0.9600 \\
$\mathrm{~N} 1-\mathrm{C} 1$ & $1.466(5)$ & $\mathrm{C} 1-\mathrm{H} 1 \mathrm{~B}$ & 0.9600 \\
$\mathrm{C} 2-\mathrm{O} 2$ & $1.245(3)$ & $\mathrm{C} 1-\mathrm{H} 1 \mathrm{C}$ & 0.9600 \\
$\mathrm{C} 2-\mathrm{N} 3$ & $1.359(4)$ & $\mathrm{N} 4-\mathrm{H} 4 \mathrm{~B}$ & 0.8600 \\
$\mathrm{~N} 3-\mathrm{C} 4$ & $1.342(3)$ & $\mathrm{N} 4-\mathrm{H} 4 \mathrm{~A}$ & 0.8600 \\
$\mathrm{C} 4-\mathrm{N} 4$ & $1.333(4)$ & $\mathrm{C} 5-\mathrm{H} 5$ & 0.9300 \\
$\mathrm{C} 4-\mathrm{C} 5$ & $1.417(4)$ & $\mathrm{C} 6-\mathrm{H} 6$ & 0.9300 \\
$\mathrm{C} 5-\mathrm{C} 6$ & $1.370(7)$ & $\mathrm{C} 1^{\prime}-\mathrm{H} 1^{\prime} 3$ & 0.9600 \\
$\mathrm{~N} 1^{\prime}-\mathrm{C} 2^{\prime}$ & $1.373(4)$ & $\mathrm{C} 1^{\prime}-\mathrm{H} 1^{\prime} 2$ & 0.9600 \\
$\mathrm{~N} 1^{\prime}-\mathrm{C} 6^{\prime}$ & $1.389(9)$ & $\mathrm{C} 1^{\prime}-\mathrm{H} 1^{\prime} 1$ & 0.9600 \\
$\mathrm{~N} 1^{\prime}-\mathrm{C} 1^{\prime}$ & $1.459(6)$ & $\mathrm{N} 3^{\prime}-\mathrm{H} 3^{\prime}$ & $0.82(4)$ \\
$\mathrm{C} 2^{\prime}-\mathrm{O} 2^{\prime}$ & $1.219(3)$ & $\mathrm{N} 4^{\prime}-\mathrm{H} 4^{\prime} 2$ & 0.8600 \\
$\mathrm{C} 2^{\prime}-\mathrm{N} 3^{\prime}$ & $1.368(4)$ & $\mathrm{N} 4^{\prime}-\mathrm{H} 4^{\prime} 1$ & 0.8600 \\
$\mathrm{~N} 3^{\prime}-\mathrm{C} 4^{\prime}$ & $1.355(3)$ & $\mathrm{C} 5^{\prime}-\mathrm{H} 5^{\prime}$ & 0.9300 \\
$\mathrm{C} 4^{\prime}-\mathrm{N} 4^{\prime}$ & $1.291(5)$ & $\mathrm{C} 6^{\prime}-\mathrm{H} 6^{\prime}$ & 0.9300 \\
$\mathrm{C} 4^{\prime}-\mathrm{C} 5^{\prime}$ & $1.426(4)$ & &
\end{tabular}




\begin{tabular}{|c|c|c|c|}
\hline $\mathrm{C} 6-\mathrm{N} 1-\mathrm{C} 2$ & $121.4(4)$ & $\mathrm{H} 1^{\prime} 1-\mathrm{C}^{\prime}-\mathrm{H} 1^{\prime} 3$ & 109.00 \\
\hline $\mathrm{C} 6-\mathrm{N} 1-\mathrm{C} 1$ & $120.6(3)$ & $\mathrm{H} 1^{\prime} 2-\mathrm{C} 1^{\prime}-\mathrm{H} 1^{\prime} 3$ & 109.00 \\
\hline $\mathrm{C} 2-\mathrm{N} 1-\mathrm{C} 1$ & $118.0(3)$ & $\mathrm{C} 2^{\prime}-\mathrm{N} 3^{\prime}-\mathrm{H} 3^{\prime}$ & $113(2)$ \\
\hline $\mathrm{O} 2-\mathrm{C} 2-\mathrm{N} 3$ & $122.3(3)$ & $\mathrm{C} 4^{\prime}-\mathrm{C} 5^{\prime}-\mathrm{H} 5^{\prime}$ & 121.00 \\
\hline $\mathrm{O} 2-\mathrm{C} 2-\mathrm{N} 1$ & $118.6(3)$ & $\mathrm{C} 4^{\prime}-\mathrm{N} 3^{\prime}-\mathrm{H} 3^{\prime}$ & $122(2)$ \\
\hline $\mathrm{N} 3-\mathrm{C} 2-\mathrm{N} 1$ & $119.2(3)$ & $\mathrm{C} 6^{\prime}-\mathrm{C} 5^{\prime}-\mathrm{H} 5^{\prime}$ & 121.00 \\
\hline $\mathrm{C} 4-\mathrm{N} 3-\mathrm{C} 2$ & $119.6(2)$ & $\mathrm{C} 4^{\prime}-\mathrm{N} 4^{\prime}-\mathrm{H} 4^{\prime} 1$ & 120.00 \\
\hline $\mathrm{N} 4-\mathrm{C} 4-\mathrm{N} 3$ & $117.9(3)$ & $\mathrm{C} 5^{\prime}-\mathrm{C} 6^{\prime}-\mathrm{H} 6^{\prime}$ & 119.00 \\
\hline $\mathrm{N} 4-\mathrm{C} 4-\mathrm{C} 5$ & $119.9(2)$ & $\mathrm{C} 4^{\prime}-\mathrm{N} 4^{\prime}-\mathrm{H} 4^{\prime} 2$ & 120.00 \\
\hline $\mathrm{N} 3-\mathrm{C} 4-\mathrm{C} 5$ & $122.2(3)$ & $\mathrm{N} 1^{\prime}-\mathrm{C} 6^{\prime}-\mathrm{H} 6^{\prime}$ & 119.00 \\
\hline $\mathrm{C} 6-\mathrm{C} 5-\mathrm{C} 4$ & $116.5(3)$ & $\mathrm{H} 4^{\prime} 1-\mathrm{N} 4^{\prime}-\mathrm{H} 4^{\prime} 2$ & 120.00 \\
\hline $\mathrm{N} 1-\mathrm{C} 6-\mathrm{C} 5$ & $121.2(3)$ & $\mathrm{H} 4 \mathrm{~A}-\mathrm{N} 4-\mathrm{H} 4 \mathrm{~B}$ & 120.00 \\
\hline $\mathrm{C} 2^{\prime}-\mathrm{N} 1^{\prime}-\mathrm{C} 6^{\prime}$ & $120.0(4)$ & $\mathrm{C} 4-\mathrm{N} 4-\mathrm{H} 4 \mathrm{~B}$ & 120.00 \\
\hline $\mathrm{C} 2^{\prime}-\mathrm{N} 1^{\prime}-\mathrm{C} 1^{\prime}$ & $118.4(3)$ & $\mathrm{C} 4-\mathrm{N} 4-\mathrm{H} 4 \mathrm{~A}$ & 120.00 \\
\hline $\mathrm{C} 6^{\prime}-\mathrm{N} 1^{\prime}-\mathrm{C} 1^{\prime}$ & $121.5(3)$ & $\mathrm{N} 1-\mathrm{C} 1-\mathrm{H} 1 \mathrm{~A}$ & 109.00 \\
\hline $\mathrm{O} 2^{\prime}-\mathrm{C} 2^{\prime}-\mathrm{N} 3^{\prime}$ & $121.8(3)$ & $\mathrm{N} 1-\mathrm{C} 1-\mathrm{H} 1 \mathrm{~B}$ & 109.00 \\
\hline $\mathrm{O} 2^{\prime}-\mathrm{C} 2^{\prime}-\mathrm{N} 1^{\prime}$ & $121.7(4)$ & $\mathrm{N} 1-\mathrm{C} 1-\mathrm{H} 1 \mathrm{C}$ & 109.00 \\
\hline $\mathrm{N} 3^{\prime}-\mathrm{C} 2^{\prime}-\mathrm{N} 1^{\prime}$ & $116.5(3)$ & $\mathrm{H} 1 \mathrm{~A}-\mathrm{C} 1-\mathrm{H} 1 \mathrm{~B}$ & 109.00 \\
\hline $\mathrm{C} 4^{\prime}-\mathrm{N} 3^{\prime}-\mathrm{C} 2^{\prime}$ & $125.1(3)$ & $\mathrm{H} 1 \mathrm{~A}-\mathrm{C} 1-\mathrm{H} 1 \mathrm{C}$ & 109.00 \\
\hline $\mathrm{N} 4^{\prime}-\mathrm{C} 4^{\prime}-\mathrm{N} 3^{\prime}$ & $119.7(3)$ & $\mathrm{H} 1 \mathrm{~B}-\mathrm{C} 1-\mathrm{H} 1 \mathrm{C}$ & 109.00 \\
\hline $\mathrm{N} 4^{\prime}-\mathrm{C} 4^{\prime}-\mathrm{C} 5^{\prime}$ & $123.2(3)$ & $\mathrm{C} 4-\mathrm{C} 5-\mathrm{H} 5$ & 122.00 \\
\hline $\mathrm{N} 3^{\prime}-\mathrm{C} 4^{\prime}-\mathrm{C} 5^{\prime}$ & $117.0(3)$ & $\mathrm{C} 6-\mathrm{C} 5-\mathrm{H} 5$ & 122.00 \\
\hline $\mathrm{C} 6^{\prime}-\mathrm{C} 5^{\prime}-\mathrm{C} 4^{\prime}$ & $118.6(3)$ & $\mathrm{N} 1^{\prime}-\mathrm{C} 1^{\prime}-\mathrm{H} 1^{\prime} 1$ & 109.00 \\
\hline $\mathrm{C} 5^{\prime}-\mathrm{C} 6^{\prime}-\mathrm{N} 1^{\prime}$ & $122.8(3)$ & $\mathrm{N} 1-\mathrm{C} 6-\mathrm{H} 6$ & 119.00 \\
\hline $\mathrm{N} 1^{\prime}-\mathrm{C} 1^{\prime}-\mathrm{H} 1^{\prime} 3$ & 109.00 & $\mathrm{~N} 1^{\prime}-\mathrm{C} 1^{\prime}-\mathrm{H} 1^{\prime} 2$ & 110.00 \\
\hline $\mathrm{H} 1^{\prime} 1-\mathrm{C} 1^{\prime}-\mathrm{H} 1^{\prime} 2$ & 109.00 & $\mathrm{C} 5-\mathrm{C} 6-\mathrm{H} 6$ & 119.00 \\
\hline $\mathrm{C} 6-\mathrm{N} 1-\mathrm{C} 2-\mathrm{O} 2$ & $-177.1(3)$ & $\mathrm{C} 6^{\prime}-\mathrm{N} 1^{\prime}-\mathrm{C} 2^{\prime}-\mathrm{O} 2^{\prime}$ & $177.5(3)$ \\
\hline $\mathrm{C} 1-\mathrm{N} 1-\mathrm{C} 2-\mathrm{O} 2$ & $4.6(5)$ & $\mathrm{C} 1^{\prime}-\mathrm{N} 1^{\prime}-\mathrm{C} 2^{\prime}-\mathrm{O} 2^{\prime}$ & $-6.9(5)$ \\
\hline $\mathrm{C} 6-\mathrm{N} 1-\mathrm{C} 2-\mathrm{N} 3$ & $2.0(5)$ & $\mathrm{C} 6^{\prime}-\mathrm{N} 1^{\prime}-\mathrm{C} 2^{\prime}-\mathrm{N} 3^{\prime}$ & $-1.2(5)$ \\
\hline $\mathrm{C} 1-\mathrm{N} 1-\mathrm{C} 2-\mathrm{N} 3$ & $-176.4(3)$ & $\mathrm{C} 1^{\prime}-\mathrm{N} 1^{\prime}-\mathrm{C} 2^{\prime}-\mathrm{N} 3^{\prime}$ & $174.4(3)$ \\
\hline $\mathrm{O} 2-\mathrm{C} 2-\mathrm{N} 3-\mathrm{C} 4$ & $176.3(3)$ & $\mathrm{O} 2^{\prime}-\mathrm{C} 2^{\prime}-\mathrm{N} 3^{\prime}-\mathrm{C} 4^{\prime}$ & $-177.4(3)$ \\
\hline $\mathrm{N} 1-\mathrm{C} 2-\mathrm{N} 3-\mathrm{C} 4$ & $-2.7(4)$ & $\mathrm{N} 1^{\prime}-\mathrm{C} 2^{\prime}-\mathrm{N} 3^{\prime}-\mathrm{C} 4^{\prime}$ & $1.3(5)$ \\
\hline $\mathrm{C} 2-\mathrm{N} 3-\mathrm{C} 4-\mathrm{N} 4$ & $-178.3(3)$ & $\mathrm{C} 2^{\prime}-\mathrm{N} 3^{\prime}-\mathrm{C} 4^{\prime}-\mathrm{N} 4^{\prime}$ & $-179.8(4)$ \\
\hline $\mathrm{C} 2-\mathrm{N} 3-\mathrm{C} 4-\mathrm{C} 5$ & $1.6(4)$ & $\mathrm{C} 2^{\prime}-\mathrm{N} 3^{\prime}-\mathrm{C} 4^{\prime}-\mathrm{C} 5^{\prime}$ & $0.5(5)$ \\
\hline $\mathrm{N} 4-\mathrm{C} 4-\mathrm{C} 5-\mathrm{C} 6$ & $-179.8(3)$ & $\mathrm{N} 4^{\prime}-\mathrm{C} 4^{\prime}-\mathrm{C} 5^{\prime}-\mathrm{C} 6^{\prime}$ & $177.8(4)$ \\
\hline $\mathrm{N} 3-\mathrm{C} 4-\mathrm{C} 5-\mathrm{C} 6$ & $0.3(5)$ & $\mathrm{N} 3^{\prime}-\mathrm{C} 4^{\prime}-\mathrm{C} 5^{\prime}-\mathrm{C} 6^{\prime}$ & $-2.4(5)$ \\
\hline $\mathrm{C} 2-\mathrm{N} 1-\mathrm{C} 6-\mathrm{C} 5$ & $0.0(6)$ & $\mathrm{C} 4^{\prime}-\mathrm{C} 5^{\prime}-\mathrm{C} 6^{\prime}-\mathrm{N} 1^{\prime}$ & $2.6(6)$ \\
\hline $\mathrm{C} 1-\mathrm{N} 1-\mathrm{C} 6-\mathrm{C} 5$ & $178.3(4)$ & $\mathrm{C} 2^{\prime}-\mathrm{N} 1^{\prime}-\mathrm{C} 6^{\prime}-\mathrm{C} 5^{\prime}$ & $-0.8(6)$ \\
\hline $\mathrm{C} 4-\mathrm{C} 5-\mathrm{C} 6-\mathrm{N} 1$ & $-1.2(6)$ & $\mathrm{C} 1^{\prime}-\mathrm{N} 1^{\prime}-\mathrm{C} 6^{\prime}-\mathrm{C} 5^{\prime}$ & $-176.2(4)$ \\
\hline
\end{tabular}

Hydrogen-bond geometry $\left(A,{ }^{\circ}\right)$

\begin{tabular}{lllll}
\hline$D-\mathrm{H}^{\cdots} \cdots A$ & $D-\mathrm{H}$ & $\mathrm{H} \cdots A$ & $D \cdots A$ & $D-\mathrm{H}^{\cdots} \cdot A$ \\
\hline $\mathrm{N} 3^{\prime}-\mathrm{H} 3^{\prime} \cdots \mathrm{N} 3$ & $0.82(4)$ & $1.99(4)$ & $2.808(4)$ & $171(4)$ \\
$\mathrm{N} 4{ }^{\prime}-\mathrm{H} 4{ }^{\prime} 1 \cdots \mathrm{O} 2$ & 0.86 & 1.92 & $2.762(4)$ & 166 \\
$\mathrm{~N} 4-\mathrm{H} 4 A^{\cdots} \cdots \mathrm{O} 2^{\prime}$ & 0.86 & 2.07 & $2.901(3)$ & 161
\end{tabular}


$\mathrm{N} 4-\mathrm{H} 4 B \cdots \mathrm{I} 1$

$\mathrm{N} 4^{\prime}-\mathrm{H} 4^{\prime} 2 \cdots \mathrm{I}^{\mathrm{i}}$

$\mathrm{C} 5-\mathrm{H} 5 \cdots \mathrm{O} 2^{\prime \text { ii }}$

$\mathrm{C} 5^{\prime}-\mathrm{H} 5^{\prime} \cdots \mathrm{O} 2^{\mathrm{iii}}$ $\begin{array}{ll}0.86 & 2.87\end{array}$

0.86

0.93

0.93
2.78

2.47

2.48
3.717 (2)

$3.633(2)$

3.088 (4)

3.085 (4)
169

171

124

123

Symmetry codes: (i) $x-1, y, z-1$; (ii) $-x+1, y+1 / 2,-z+1$; (iii) $-x, y-1 / 2,-z$. 